\title{
Teratoma formation of human embryonic stem cells in three-dimensional perfusion culture bioreactors
}

\author{
H. Stachelscheid ${ }^{1 *}$, A. Wulf-Goldenberg ${ }^{2}$, K. Eckert $^{2}$, J. Jensen ${ }^{3}$, J. Edsbagge ${ }^{3}$, P. Björquist ${ }^{3}$, M. Rivero ${ }^{3}$, \\ R. Strehl ${ }^{3}$, J. Jozefczuk ${ }^{4}$, A. Prigione ${ }^{4}$, J. Adjaye ${ }^{4}$, T. Urbaniak ${ }^{1}$, P. Bussmann ${ }^{1}$, K. Zeilinger ${ }^{1}$ and \\ J. C. Gerlach ${ }^{5}$ \\ ${ }^{1}$ Berlin-Brandenburg Center for Regenerative Therapies, Charité-Universitätsmedizin Berlin, Germany \\ ${ }^{2}$ Max Delbrück Centre for Molecular Medicine, Berlin, Germany \\ ${ }^{3}$ Cellartis AB, Göteborg, Sweden \\ ${ }^{4}$ Max Planck Institute for Molecular Genetics, Berlin, Germany \\ ${ }^{5}$ McGowan Institute for Regenerative Medicine Departments of Surgery and Bioengineering, University of Pittsburgh, PA, USA
}

\begin{abstract}
Teratoma formation in mice is today the most stringent test for pluripotency that is available for human pluripotent cells, as chimera formation and tetraploid complementation cannot be performed with human cells. The teratoma assay could also be applied for assessing the safety of human pluripotent cell-derived cell populations intended for therapeutic applications. In our study we examined the spontaneous differentiation behaviour of human embryonic stem cells (hESCs) in a perfused 3D multi-compartment bioreactor system and compared it with differentiation of hESCs and human induced pluripotent cells (hiPSCs) cultured in vitro as embryoid bodies and in vivo in an experimental mouse model of teratoma formation. Results from biochemical, histological/immunohistological and ultrastuctural analyses revealed that hESCs cultured in bioreactors formed tissue-like structures containing derivatives of all three germ layers. Comparison with embryoid bodies and the teratomas revealed a high degree of similarity of the tissues formed in the bioreactor to these in the teratomas at the histological as well as transcriptional level, as detected by comparative whole-genome RNA expression profiling. The 3D culture system represents a novel in vitro model that permits stable long-term cultivation, spontaneous multi-lineage differentiation and tissue formation of pluripotent cells that is comparable to in vivo differentiation. Such a model is of interest, e.g. for the development of novel cell differentiation strategies. In addition, the 3D in vitro model could be used for teratoma studies and pluripotency assays in a fully defined, controlled environment, alternatively to in vivo mouse models. Copyright (c) 2012 John Wiley \& Sons, Ltd.
\end{abstract}

Received 13 April 2011; Revised 14 October 2011; Accepted 5 January 2012

Supporting information may be found in the online version of this article.

Keywords human embryonic stem cells; bioreactor; in vitro model; 3D tissue formation; differentiation; teratoma; embryoid body

\section{Introduction}

Human embryonic stem cells (hESCs) are pluripotent cells capable of unlimited self-renewal and differentiation into cells representative of all three embryonic germ layers.

*Correspondence to: H. Stachelscheid, Charité-Universitätsmedizin Berlin, Berlin-Brandenburg Center for Regenerative Therapies, Augustenburger Platz 1, 13353 Berlin, Germany. E-mail: Harald. Stachelscheid@charite.de
They hold large potential as a cell source for applications in basic science, pharmacology, toxicology and cell-based therapy development in regenerative medicine.

When transplanted into immune-compromised animals, undifferentiated hESCs form teratomas, consisting of different tissue types derived from all three germ layers (Przyborski, 2005; Blum and Benvenisty, 2008). In human stem cell research, teratoma formation in mice is used as an important in vivo pluripotency assay, in conjunction with analysis of pluripotency marker expression and in vitro differentiation assays. It is the most stringent method available 
for testing the developmental potential of isolated and cultured hESCs or other human pluripotent cells, such as human induced pluripotent stem cells (hiPSCs), since germ line transmission as applied for testing the pluripotency of mouse ES cells is prohibited in hESCs research, for ethical reasons (Heins et al., 2004; Valbuena et al., 2006; Adewumi et al., 2007). In addition to this, teratomas represent an alternative development model because developmental processes cannot be investigated in intact mammalian embryos. Teratomas exhibit arrangements of different tissue types that in many ways recapitulate organogenesis within the embryo (Przyborski, 2005). Studying teratomas could result in a better understanding of the stepwise developmental processes and their molecular bases and might provide useful information for the development of tissueengineering technologies (Aleckovic and Simon, 2008).

The teratoma assay could also be applied for assessing the safety of human pluripotent stem cell-derived cell populations intended for therapeutic applications, because small quantities of undifferentiated cells contaminating any transplant material could be efficiently detected by their capacity for multi-lineage differentiation. Currently, however, there is no in vitro model for teratoma formation available, therefore to reduce animal testing the development of new methods is of great interest.

Flat plastic dishes of various formats are the most commonly used in vitro culture systems. These represent static two-dimensional (2D) open-batch systems with discontinuous medium exchange. Batch systems are characterized by periodical changes of the culture environment, such as metabolite accumulation and nutrient decrease in the culture medium between medium changes. Despite efforts to stimulate cell aggregate formation by coating the 2D surface with biomatrices, these systems are limited in supporting the generation of 3D tissue structures by stem cells.

In suspension, hESCs form multicellular aggregates, in which the cells start to differentiate spontaneously. The formation of these so-called embryoid bodies (EBs) is a wellestablished hESC/hiPSC culture method (Itskovitz-Eldor et al., 2000) considering three-dimensional (3D) techniques, but under static conditions. EBs consist of differentiated and undifferentiated cells that can be cavitated and cystic. They are thought to resemble early post-implantation embryos and frequently progress through a series of differentiation stages (Desbaillets et al., 2000; Vallier and Pedersen, 2005). EB culture can be performed until the EBs start showing central necrosis, due to the limited mass exchange in the body. The use of 3D perfusion culture models provides a promising option for in vitro teratoma generation from pluripotent stem cells, since they approximate the physiological situation of the cells within the organism more closely. We previously developed a four-compartment capillary membrane technology for dynamic perfusion culture of primary cells and stem cells, with integral gas exchange and decentralized medium supply avoiding nonphysiological gradients (Gerlach et al., 2003). Two interwoven independently perfused capillary beds provide a more homogeneous 3D environment and a third interwoven oxygenation capillary compartment facilitates obtaining cell densities similar to those in natural tissues. Initial studies on the culture of mouse embryonic stem cells in the bioreactors enabled controllable expansion or differentiation of the cells, and showed the possibility of scaling up the technology (Gerlach et al., 2010a, 2010b). The aim of this study was to demonstrate the suitability of the bioreactor system for establishing a 3D in vitro model for tissue formation by hESCs. The growth and differentiation behaviour of hESCs was examined in long-term culture experiments by the analysis of metabolic parameters in the culture medium, RNA expression profiling using microarrays, expression of differentiation markers, histology and transmission electron microscopy. The results were compared with those of hiPSCs and hESCs cultured as embryoid bodies and an in vivo teratoma model in immunodeficient NOD/SCID mice. In addition, the influences of co-cultivation with feeder cells and of the culture medium used were examined.

\section{Materials and methods}

\section{1. hESCs expansion}

The human embryonic stem cell line SA002 (Heins et al., 2004) was provided by Cellartis (Gothenburg, Sweden). Larger-scale cell expansion for bioreactor inoculation was performed using the single-cell enzymatic dissociation (SCED) culture system, as described by Ellerstrom et al. (2007). Briefly, hESCs were cultured in VitroHES medium (Vitrolife AB, Kungsbacka, Sweden; http:// www.vitrolife.com) supplemented with $10 \mathrm{ng} / \mathrm{ml}$ human recombinant basic fibroblast growth factor (bFGF; PeproTec) in culture dishes containing a high-density human foreskin fibroblasts (HFFs) layer. VitroHES was composed as previously described (Amit et al., 2000; Heins et al., 2004). Upon 80-90\% confluence, the hESCs were enzymatically dissociated, using TrypLE Select (Invitrogen), and seeded onto fresh HFFs at split ratios between 1:4 and $1: 10$.

\subsection{The investigated bioreactor}

The multicompartment bioreactors used for this study are composed of three independent, yet interwoven, hollow-fibre capillary membrane systems (Figure 1) that are integrated into a two-component polyurethane housing (PUR; Morton, Germany). Two hydrophilic capillary systems for medium perfusion are made of microporous polyethersulphone capillary membranes with a molecular weight (MW) cut-off of approximately 500000 (mPES; Membrana, Germany). The third system is made of hydrophobic multilaminate hollow-fibre membrane capillaries (MHF; Mitsubishi, Tokyo, Japan) to enable gas exchange. The cells located within the extracapillary space (cell compartment) are thus exposed to a decentralized medium supply with high mass exchange rates and direct membrane oxygenation via diffusion (for illustrations and 
technical descriptions, see Gerlach et al., 2010a, 2010b). The bioreactor is integrated into a processor-controlled perfusion device with pressure and flow regulation for modular pumps, with exchangeable multichannel flowheads and gears for medium recirculation and substitution. A heating unit provides a constant temperature within the perfusion circuit. The flow rates of air and $\mathrm{CO}_{2}$ were controlled using integrated rotameters. Perfusion tubings with bubble traps were made from standard medical-grade dialysis PVC (B. Braun, Melsungen, Germany). Sterilization was performed with ethylene oxide or formaldehyde gas, followed by a degassing period of at least 7 days.

\subsection{Bioreactor cultures}

Before initial cell inoculation bioreactors underwent a conditioning phase of $24-72 \mathrm{~h}$ with recirculation of medium, after which cell inoculation cultures were perfused at a flow rate of $22-30 \mathrm{ml} / \mathrm{min}$. The bioreactors were kept at $37^{\circ} \mathrm{C}$. The flow of the air/ $\mathrm{CO}_{2}$ mixture in the gas compartment was maintained at $40 \mathrm{ml} / \mathrm{min}$. The $\mathrm{pH}$, partial pressures of oxygen $\left(\mathrm{pO}_{2}\right)$ and carbon dioxide $\left(p \mathrm{CO}_{2}\right)$ and the acid/base status were periodically measured (ABL 5, Radio Meter Copenhagen, Copenhagen, Denmark) and the air: $\mathrm{CO}_{2}$ mixing ratio was adjusted to maintain a stable $\mathrm{pH}$ of 7.2-7.3.

hESCs were cultured with or without co-inoculation of inactivated HFFs using VitroHES medium (Vitrolife) supplemented with $10 \mathrm{ng} / \mathrm{ml} \mathrm{bFGF}$ and $50 \mu \mathrm{g} / \mathrm{ml}$ gentamycin (Biochrom) or Dulbecco's modified Eagle's medium (DMEM)/F12 supplemented with 20\% fetal calf serum (FCS), 1\% non-essential amino acids (NEAAs), $50 \mu \mathrm{g} / \mathrm{ml}$ gentamycin (all Biochrom) and 1\% GlutaMax-I (Invitrogen) as the culture medium. Table 1 lists the experiments performed and the experimental conditions. After cell inoculation, fresh medium was continuously added to the perfusion circuit, initially at a flow rate of $2 \mathrm{ml} / \mathrm{h}$. The fresh medium feed in all bioreactor experiments was adjusted considering the parameters measured in the culture medium to maintain stable glucose levels, prevent accumulation of toxic metabolic cell products and maintain a stable medium osmolality.

\subsection{Metabolic parameters in the perfusion medium}

The metabolic activity and differentiation of the cells inside the bioreactors were characterized on a daily basis by measuring soluble factors in the medium outflow and in the recirculating medium. The following parameters were measured, using automated clinical chemistry analysers (Roche Diagnostics, Germany): $\alpha$-fetoprotein, alkaline phosphatase, alanine transaminase, aspartate transaminase, $\beta$-human chorionic gonadotropin, c-peptide, carcinoembryonic antigen, cytokeratin fragment 19, erythropoetin, oestradiol, follicle-stimulating hormone, $\gamma$-glutamyltransferase, glucose, lactate, lactate dehydrogenase, luteinizing hormone, neuronspecific enolase, osmolality, osteocalcin, pseudocholinesterase, progesterone, prolactin, S-100, thyroid-stimulating hormone, tissue plasminogen activator and transferrin. Additionally glutamine, glutamate, glucose, lactate, ammonium, $\mathrm{pH}$, sodium and potassium were measured using a BioProfile 100 Plus device (Nova Biomedical, USA). Activin A and insulin were measured by enzyme-linked immunosorbent assays (ELISAs), following the manufacturer's recommendations (activin A using products DY338, DY999, DY994, DY995, from R\&D Systems, Wiesbaden-Nordenstadt, Germany; insulin using an ELISA from Invitrogen).

The production or consumption rate of each substance was calculated per bioreactor per day by multiplying the

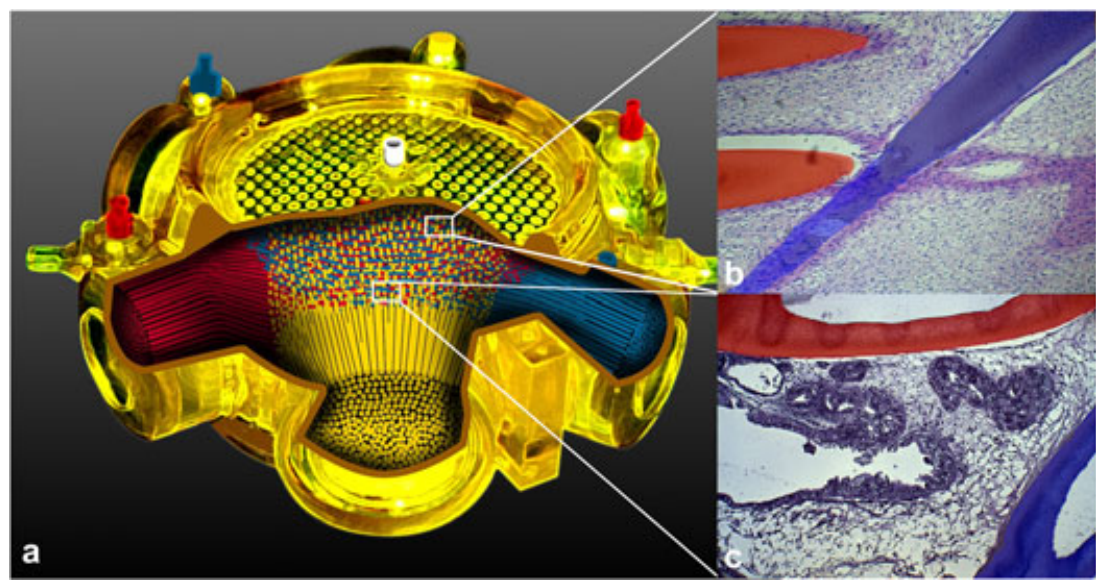

Figure 1. Bioreactor design. (a) Housing with tube connectors, partially opened to view the capillary systems. All membrane compartments are interwoven, forming a tight network. The smallest unit formed by interweaving is framed by two medium capillaries (medium perfusion compartments; red and blue) and two gas capillaries (oxygenation compartment; yellow). This allows scale-up without changing the smallest units. The corresponding capillaries of each compartment are bundled to common in- and outflow heads to be connected to tube systems. Each capillary membrane compartment can be perfused independently. (b, c) The cells are located within the extracapillary space [cell compartment, exemplary pictures show histological sections from fibroblasts (b) and differentiated hESCs (c) in the bioreactor]. The cell aggregate size in the cell compartment is limited by the space between the capillaries with average intercapillary distances of $500 \mu \mathrm{m}$. Cells are inoculated into the cell compartment via open-ending tubes, which allows distribution of the injected cells within the cell compartment (not shown) 
Table 1. Overview of the performed bioreactor experiments

\begin{tabular}{llclc}
\hline Experiment name & \multicolumn{1}{c}{ Cell types } & $\begin{array}{c}\text { Inoculated cell } \\
\text { numbers }\end{array}$ & Medium & $\begin{array}{c}\text { Length of bioreactor } \\
\text { run (days) }\end{array}$ \\
\hline hESC-A & hESC HFF (irradiated) & $5.7 \times 10^{7} 4.2 \times 10^{7}$ & VitroHES $+10 \mathrm{ng} / \mathrm{ml} \mathrm{bFGF}$ & 50 \\
hESC-B & hESC HFF (irradiated) & $4.5 \times 10^{7} 4.6 \times 10^{7}$ & VitroHES + 10 ng/ml bFGF & 50 \\
hESC-C & hESC & $15.9 \times 10^{7}$ & VitroHES + 10 ng/ml bFGF & 50 \\
hESC-D & hESC & $15.6 \times 10^{7}$ & DMEM/F12 + 20\% FCS + Glutamax + NEAA & 50 \\
\hline
\end{tabular}

actual waste volume of the day by the difference between the substance concentration in the feed medium and that in the waste medium.

\subsection{Sample acquisition from the bioreactor}

At the end of the scheduled culture period, the bioreactors were shut down and the tubing was disconnected. The lower bioreactor lid was opened and samples of the cell mass, including the capillary layers, were cut out using sterile scalpels and forceps for further analysis. For histological analysis, samples were directly fixed and embedded as described below.

\subsection{In vivo teratoma formation}

All animal experiments were performed under the guidelines of the German Animal Protection Law, with the approval of the responsible local authorities (Landesamt für Gesundheit und Soziales Berlin; G 0221/03). Into each recipient NOD/SCID mouse, an aliquot of $1-3 \times 10^{6}$ cells from the cell suspension used for bioreactor inoculation was injected subcutaneously within a Matrigel (BD Biosciences) suspension. Body weights and tumour sizes were measured once a week. For in vivo experiments the same time schedule as for bioreactor experiments was applied. The tumours were excised and used for RNA expression profiling and histological analysis.

\subsection{Embryoid body formation}

Embryoid bodies (EBs) were generated by seeding hESCs and hiPSCs harvested as clusters onto low-attachment dishes in VitroHES medium without bFGF supplementation. At the time-points days 6 and 20, the EBs were harvested and fixed in 4\% buffered formaldehyde solution, embedded in paraffin and cut into $2 \mu \mathrm{m}$ sections. Haematoxtylin and eosin (H\&E) staining was performed according to standard laboratory procedures.

\subsection{Expression profiling}

To isolate RNA from cells/tissue cultured in the bioreactor, excised capillaries were washed with PBS without $\mathrm{CaMg}$ and incubated for $3 \mathrm{~min}$ in $0.05-0.02 \%$ trypsinEDTA solution (Biochrom). Total RNA was isolated using the RNeasy Kit (Qiagen, Hilden, Germany), following the manufacturer's protocol. The teratomas were disrupted using a TissueLyser II, followed by homogenization with a QIAshredder (both Quiagen). Single cells were lysed by direct addition of lysis buffer to the cell pellet. Biotin-labelled cRNA was generated using the Illumina TotalPrep RNA Amplification Kit (Ambion, USA) with 300 ng quality-checked total RNA as input. cRNA samples were hybridized on Illumina human-8v2 BeadChips, which harbour ca. 24,000 RefSeq transcripts (Kuhn et al., 2004). In addition to the data generated in this study, RNA expression profile data from Illumina human-8v3 BeadChips generated from EBs of two hiPSC cell lines (iPS2 and iPS4; Prigione et al., 2010) and two hESCs lines (H1 and H9; WiCell Institute, Madison, WI, USA) that were formed using comparable culture conditions (Prigione and Adjaye, 2010) were included in the data analysis. Data analysis was performed using BeadStudio v 3 software (Illumina), MultiExperiment Viewer (MeV) (Saeed et al., 2003) and Bioconductor (Gentleman et al., 2004). Functional enrichment analysis was performed using DAVID (http://david.abcc.ncifcrf.gov).

\subsection{Histology}

Samples were fixed in 4\% buffered formaldehyde solution, embedded in paraffin and cut into $5 \mu \mathrm{m}$ sections. H\&E staining was performed according to standard laboratory procedures.

The following primary antibodies were used for immunofluorescence staining: monoclonal mouse anti-human smooth muscle actin IgG2a (ASMA), monoclonal mouse anti-human desmin IgG1 (Dako, Denmark), monoclonal mouse anti-neuron-specific $\beta$ III-Tubulin IgG2a (R\&D Systems), monoclonal mouse anti nestin IgG1 (BectonDickinson), polyclonal goat anti HNF- $3 \beta$ IgG, monoclonal mouse anti OCT-4 IgG2b and polyclonal rabbit anti vimentin IgG (Santa Cruz Biotechnology). As secondary antibodies, the following polyclonal antibodies were used: goat anti-mouse IgG-Cy2, goat anti-rabbit IgG-Cy3 (Dianova), goat anti-mouse IgG2a-TRICT, goat anti-mouse IgG-FITC, goat anti-mouse IgG-FITC, goat anti-mouse IgG-Cy3, goat anti-mouse IgG-FITC (Jackson Immunoresearch Laboratories, West Grove, PA, USA) and donkey anti-goat IgG-Cy3 (Santa Cruz Biotechnology). For non-specific staining of the nuclei, sections were incubated with 4',6-diamidino-2-phenylindole. Sections were analysed using an inverse microscope (Axiovert 200M, Carl Zeiss, Germany) equipped with a CCD camera (Retiga 2000R, QImaging, Canada). The pictures were 
acquired and processed using the digital imaging software Image Pro Plus (Media Cybernetics, USA).

For transmission electron microscopy (TEM), samples were fixed with 5\% glutaraldehyde. After immersion for

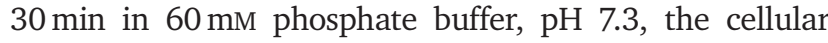
aggregates were postfixed in $2 \% \mathrm{OsO}_{4}$ for $2 \mathrm{~h}$, progressively dehydrated in ethanol and then embedded in Araldite (Serva, Germany). Ultra-thin sections were contrasted with uranyl acetate and Reynold's lead citrate (Chroma, Germany) before electron microscopic examination.

\section{Results}

\subsection{Differentiation of hESCs in the bioreactor}

Four long-term bioreactor experiments were carried out over a period of 50 days each (experiments hESC-A, hESC-B, hESC-C, hESC-D; Table 1) and compared to 11 in vivo teratoma assays. To analyse the impact of feeder cells, irradiated HFFs were inoculated into the bioreactors 2 days before inoculation of hESCs in experiments hESC-A and hESC-B, whereas experiments hESC-C and hESC-D were performed without pre-inoculation of feeder cells. The culture medium used in bioreactors hESC-A to -C contained serum replacement and $10 \mathrm{ng} / \mathrm{ml} \mathrm{bFGF}$.

In bioreactor hESC-D the culture medium was changed to a nutrient-rich culture medium containing FCS, which was chosen based on the hypothesis that such a medium better approximates to the in vivo environment of the cells when they are transplanted in a mouse teratoma formation assay and therefore will enhance differentiation. In all experiments, bioreactors were inoculated with hESC suspensions derived from $2 \mathrm{D}$ hESC/HFF co-cultures. FACS analysis of the inoculated cell suspensions showed that $45 \pm 13 \%$ of cells were positive for both OCT 4 and SSEA 4 and therefore can be assumed to represent undifferentiated hESCs. The cells that were negative for one or both pluripotency markers represent HFFs or other cell types developed from the hESCs by differentiation (see Supporting information, Supplementary methods and Figure S1).

During culture glucose, lactate and LDH concentrations were measured daily in the bioreactor perfusate and in the medium circuit outflow to evaluate the metabolic activity of the cells and to detect potential cell damage. Glucose, lactate and $\mathrm{LDH}$ showed similar time courses in bioreactors hESC-A, -B and -C, while hESC-D performed with FCScontaining nutrient-rich medium showed different time profiles of these parameters. In bioreactors hESC-A, -B and -C, a slow but constant increase of glucose consumption and lactate production was observed over the culture period, indicating cell proliferation. LDH levels decreased after an initial peak, which presumably was due to cell damage after cell inoculation (Figure 2a-c). In bioreactor hESC-D, glucose, lactate and LDH showed constant levels after an initial peak in LDH release (Figure 2d).

To identify soluble markers for potential cell differentiation processes, several factors were measured in the medium outflow. Here, interesting patterns in the concentration of $\alpha$-fetoprotein (AFP), $\beta$-human chorionic gonadotropin ( $\beta$-hCG) and activin A could be detected (Figure 2e-h). During human embryonic development, AFP is first expressed in the extraembryonic primitive endoderm (yolk sac), then in the hind- and midgut endoderm and eventually in the foregut hepatic diverticulum. Later on, AFP expression is found in the mesonephric duct and tubules and also transiently in the developing pancreas (Mizejewski, 2004). An onset of AFP production suggests differentiation of hESCs towards primitive endoderm. $\beta$-hCG represents a marker for the differentiation of hESCs towards trophectoderm (Xu et al., 2002) and is also known as a clinical marker for germ cell tumours (Stenman et al., 2006). In normal fetal development, $\beta$-hCG is produced soon after conception by the embryo, and later by the syncytiotrophoblast. The detection of $\beta$-hCG indicates the formation of cells of the trophectodermal lineage. Activin A was described to have a strong positive effect on the self-renewal of undifferentiated hESCs in vitro (Beattie et al., 2005; James et al., 2005; Vallier et al., 2005; Xiao et al., 2006). In bioreactors hESC-A and hESC-B, activin A production showed initially high concentrations, subsequently decreased and reached a minimum between days 15 and 25. This is the expected time span the inactivated HFF feeder cells survive in the bioreactors. Afterwards, activin A again slowly increased until the end of the bioreactor runs (Figure 2e, f). In bioreactor hESC-C, in which the same culture medium was used but without additional feeder cells, only a low rapidly decreasing activin A production could be detected from the beginning of the experiment until day 10 (Figure $2 \mathrm{~g}$ ). Bioreactor hESC-D run with the FCScontaining nutrient-rich medium and without feeder cells showed a decreasing activin A production that could only be detected until day 32 (Figure $2 \mathrm{~h}$ ). This suggests that in the presence of a high activin A concentration a differentiation in the bioreactor is reduced. Results from parallel 2D experiments with HFFs only showed that activin A production is stimulated by bFGF in combination with medium containing serum replacement (see Supporting information, Supplementary methods and Figure S2). This suggests that activin $\mathrm{A}$ in bioreactors hESC-A, -B and -C is produced by the HFF feeder cells that were co-inoculated or were contained in a low percentage in the inoculated hESC cell suspensions derived from the hESC/HFF co-cultures.

In all experiments, the onset of AFP and/or $\beta$-hCG production characterized ongoing cell differentiation in the bioreactor, which started as soon as the activin A concentration in the medium reached a minimum. In bioreactors hESC-A and -B, AFP production started to rise at about days $15-20$ and continued to increase during the entire observed period of 50 days. $\beta$-hCG started to increase in these bioreactors at about day 20, peaked at around day 40 and decreased thereafter (Figure 2e, f). In contrast, $\beta$-hCG production started to increase from day 10 in bioreactor hESC-C performed without feeder cells but stayed on a much lower level compared to bioreactors hESC-A and -B. Most notably, the level of AFP exhibited an exponential increase from day 10 onwards, with a peak 
of $350 \mathrm{ng} / \mathrm{h}$ on day 25 followed by a slight decrease until day 37 and a stable level until the end of the experiment (Figure $2 \mathrm{~g}$ ). In bioreactor hESC-D run with FCS-containing, nutrient-rich medium, no $\beta$-hCG production could be detected throughout the experiment duration of 50 days and only low levels of AFP were observed, starting at day 41 (Figure 2h). Comparison of the production levels of AFP and $\beta$-HCG in bioreactors hESC-A to hESC-C could indicate that the addition of HFF feeder cells enhances differentiation towards trophectoderm, while in their absence differentiation towards primitive endoderm may be favoured.

Considering the histology described below, these results show that hESCs grow and differentiate in the 3D perfusion system used and that the culture behaviour can be monitored by the analysis of soluble marker molecules in the culture perfusate.

\subsection{Histology}

The morphology of the structures formed in vitro in the bioreactors was analysed and compared with those of EBs and of in vivo teratomas formed in NOD/SCID mice by subcutaneous hESCs injection. The in vivo teratomas were grown in parallel to the bioreactor cultures and for the same time period. EBs derived from hESCs were cultured for 6 or 20 days and EBs derived from hiPSC for 6 , 20 or 50 days.

The histology of the bioreactors showed that the hESCs differentiated and formed tissue-like structures comparable to those formed in the teratomas, although the degree of differentiation differed between the individual bioreactors (Figure 3a-d, i-1). In EBs derived from hESCs and hiPSCs, a much lower level of differentiation was found compared to the bioreactor cultures and teratomas (Figure 3m-p). They consisted of a limited number of cell types; some were cavitated and even after 50 days of culture only a few tissue like structures could be observed (Figure 3m-p; see also Supporting information, Figure S4). In general, only connective tissue-like structures indicating mesodermal differentiation, areas displaying a morphology of embryonic neural rosettes, indicating ectodermal differentiation but no epithelial or glandular structures, could be observed. Additionally, in many EBs necrotic areas could be observed, mostly in the centres of the aggregates (Figure 3m, p; see also Supporting information, Figure S4h, i). Such necrotic areas could not be detected in any of the bioreactors or teratomas.

Bioreactors hESC-A to hESC-C exhibited a comparable histology regarding the number and types of different
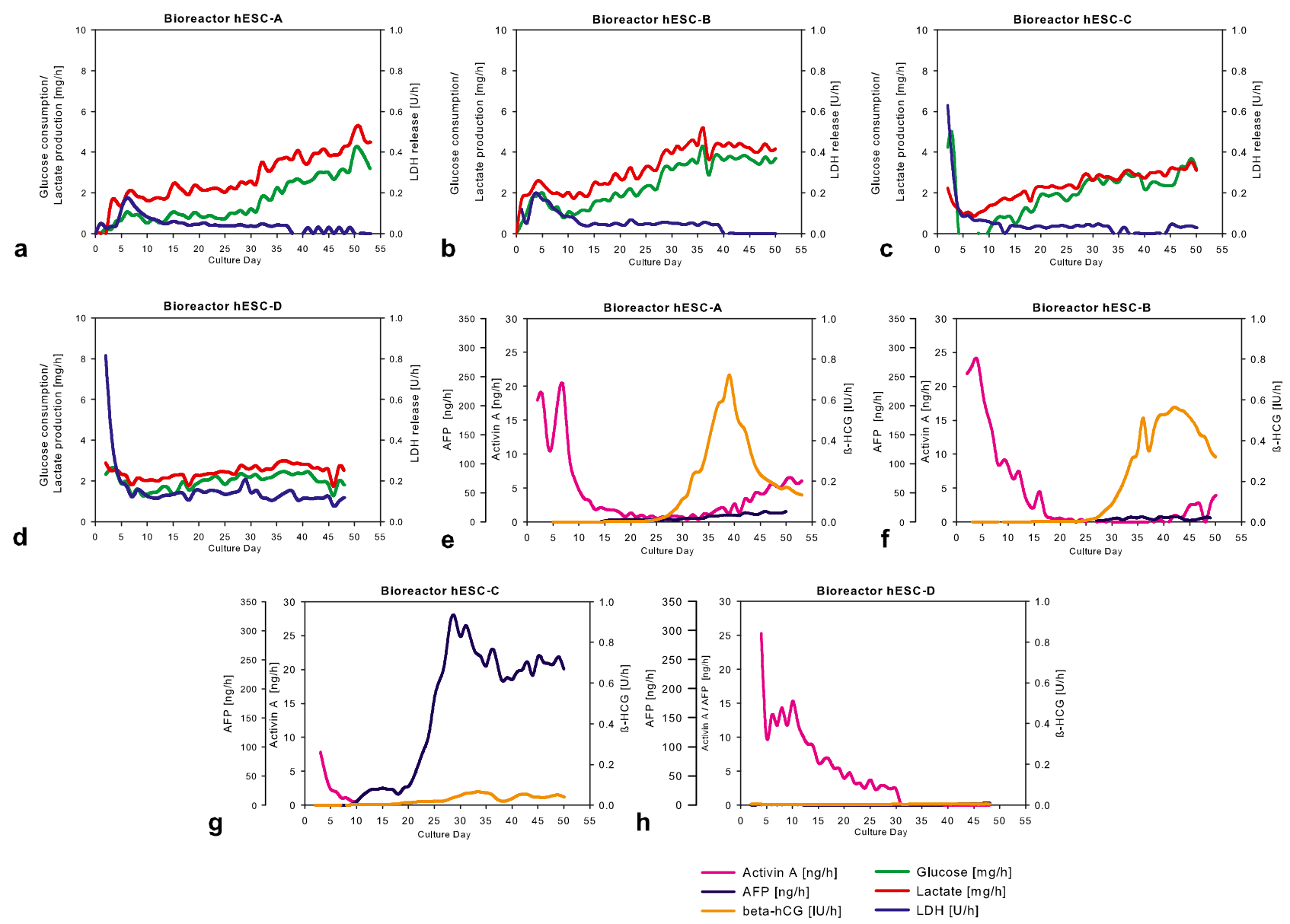

Figure 2. Time course of metabolic parameters and differentiation markers measured in the medium outflow of hESC bioreactors. Graphs (a-d) show glucose consumption (green), lactate production (red) and LHD release (blue) in the bioreactors hESCs-A, -B, -C and -D, respectively. Activin A production (pink), AFP production (dark blue) and $\beta$-hCG production (yellow) by the cells in bioreactors is shown in graphs $(\mathrm{e}-\mathrm{h})$ 
tissues that could be detected (Figure 3). In all three bioreactors, structures and typical marker expression showing differentiation into tissue of all three germ layers could be detected, similar to the in vivo teratomas. However, in comparison with the in vivo teratomas, some differences in the proportions of individual tissue types were observed. In particular, the material gained from the bioreactors contained more ectodermal structures compared to the teratomas. These structures could be identified by their morphology, mostly in the form of embryonic neural rosettes (Figure 3c, d) and the expression of the neural markers nestin (Gilyarov, 2008) and $\beta$ III-tubulin (Katsetos et al., 2003a, 2003b) (Figure 4a, b, d, e). Mesodermallyderived tissues were similarly observed in bioreactors and teratomas. In general, a frequent occurrence of connective tissue comprised of fibroblasts and collagen-rich extracellular matrix (Figure 3d, f, h, i) and also numerous clusters of hyaline cartilage (Figure 3a, e) were detected in the examined samples. The presence of mesodermal tissues was further confirmed by the immunohistochemical detection of $\alpha$-smooth muscle actin characteristic for smooth muscle cells (Figure 4c, f), type III intermediate filament desmin, which is one of the earliest protein markers for muscle tissue in embryogenesis (Bar et al., 2004) (Figure 4g, j), and vimentin as a general marker for mesenchymal cells (Figure $4 \mathrm{~h}, \mathrm{k}$ ). Endodermally-derived tissues were found frequently in the examined samples and showed a similar quantity and distribution in bioreactors and teratomas. These structures were identified by their typical morphology in the shape of epithelial (Figure 3b, j) and glandular structures (Figure 3k) and also by ultrastructural features such as bioreactor.

The histology of bioreactor hESC-D cultured without feeder cells and with FCS-containing medium differed from that of the other bioreactors. Next to connective tissue-like structures, some clusters of cells with a low cytoplasm:nucleus ratio characteristic of undifferentiated hESCs could be detected (see Supporting information, Figure S3). Many of these clusters co-expressed the pluripotency markers OCT4 and TRA-1-81, which suggested that they represented undifferentiated hESCs (see Supporting information, Figure S3). Structures showing further maturation were not observed.

\subsection{Gene expression profiling}

To compare the gene expression profiles of hESCs cultured in the bioreactors with the in vivo teratomas that were grown in parallel, whole-genome microarray profiling was performed. For comparison of the expression profiles of the bioreactor cultures and the teratomas to these of EBs formed by hESCs and hiPSCs, data generated in nother study (Prigione and Adjaye, 2010), using comparable culture conditions, were included in the data analysis. As controls, active and inactivated HFF samples from the hESC/HFF cell suspensions, which were inoculated into the bioreactors (abbreviated to SCED), and undifferentiated hESCs cut from colonies growing in co-culture with
MEF were used (for a detailed sample description, see Supporting information, Table S1).

Transcriptional similarities between the different samples were analysed by hierarchical clustering. The bioreactors that showed a similar histology and similar time courses of differentiation factors, as described above, also exhibited a similar gene expression profile. These results specifically show that the transcriptomes of bioreactors hESC-A, -B and -C are very similar and therefore cluster together (Figure 5a). This group of bioreactors was also closely related to the group of in vivo teratomas, which indicates a similar expression profile of these groups. Thus, a similar cell differentiation in these bioreactors and in the teratomas can be assumed. Interestingly, the expression profile of bioreactor hESC-C showed even more similarities to the expression profiles of the teratomas than to the expression profiles of hESC-A and $-\mathrm{B}$.

Bioreactor hESC-D, which was cultured without feeder cells and using FCS-containing medium, exhibited a transcriptome similar to that of undifferentiated hESCs (line SA002) grown in HFF and MEF 2D co-cultures. Again, the result of morphological analysis of this bioreactor that showed little to no differentiation was confirmed by the results of the gene expression analysis. Since the initially high activin A production decreased much more slowly and was maintained on a higher level compared to the other bioreactors, the results from gene expression analysis and from histological studies suggest that differentiation in this reactor was suppressed by a 'feeder cell-like' activity. This could be due to differentiation of some hESCs into fibroblasts or fibroblast-like cells with the ability to secrete activin A, and that are able to support the self-renewal of undifferentiated hESCs, as has been described in 2D cultures in the literature (Xu et al., 2004; Stojkovic et al., 2005; Yoo et al., 2005; Gonzalez et al., 2008).

The last group in the hierarchical tree consisted of the undifferentiated hESC and hiPSC lines H1, H9, iPS2 and iPS4 and the EBs derived from them. Furthermore, this group was closely related to the group of bioreactor hESC-D and to the undifferentiated cells of cell line SA002 that were used in the bioreactor and teratoma experiments. This indicates that the EBs are less differentiated than the teratomas and bioreactors and therefore cluster together with the undifferentiated pluripotent cells. Comparison of the significantly expressed genes (detection values $p \leq 0.01$ ) in the bioreactors, teratomas and EBs shows that bioreactors and teratomas show a common expression for at least five more genes than each of them with the EBs (Figure 5b). Again these results confirm the observations made at the histological level.

To further compare the expression profiles of the group of bioreactors that clustered together (hESC-A, -B and -C) with the group of in vivo teratomas, a differential expression analysis was performed. The results from the analysis showed that 12 genes were differentially expressed in the teratomas compared to 146 genes in the bioreactors, respectively (Figure $5 \mathrm{c}, \mathrm{d}$ ). The resulting gene lists are relatively 
short, which is a further indication that the two groups are closely related.

The differentially expressed genes in the bioreactors were further analysed by functional annotation analysis. The result of the functional annotation clustering performed is shown in Table S2 (see Supporting information). Most notable are the first and fourth functional groups, that contain gene ontology (GO) terms describing developmental or morphogenetic processes, and the functional groups two and nine, that contain terms describing neural differentiation.

The analysis of the expression profiles confirmed the observations obtained at the metabolic and histological levels. The transcriptomes of teratoma structures generated in the bioreactors and in vivo teratomas are similar and differ significantly from these of the embryoid bodies. There is only a very low number of genes that are differentially expressed in the bioreactors and teratomas; analysis of these genes by functional annotation clustering also indicated that neural differentiation was pronounced in the bioreactors.

\section{Discussion}

In this paper, we introduce a $3 \mathrm{D}$ perfusion bioreactor system that allows in vivo-like tissue formation by hESCs and potentially by pluripotent stem cell types from other sources. Our results from spontaneously differentiating hESCs revealed high similarities with in vivo teratomas generated in mice and a lower degree of similarity to EBs formed in suspension cultures in vitro, at the histological, ultrastructural and RNA expression levels.

In both bioreactors and teratomas, differentiated tissues of all three germ layers could be observed histologically, with some differences in the proportion of individual cell/tissue types. In particular, the relative number of neural structures was distinctly larger in the bioreactor

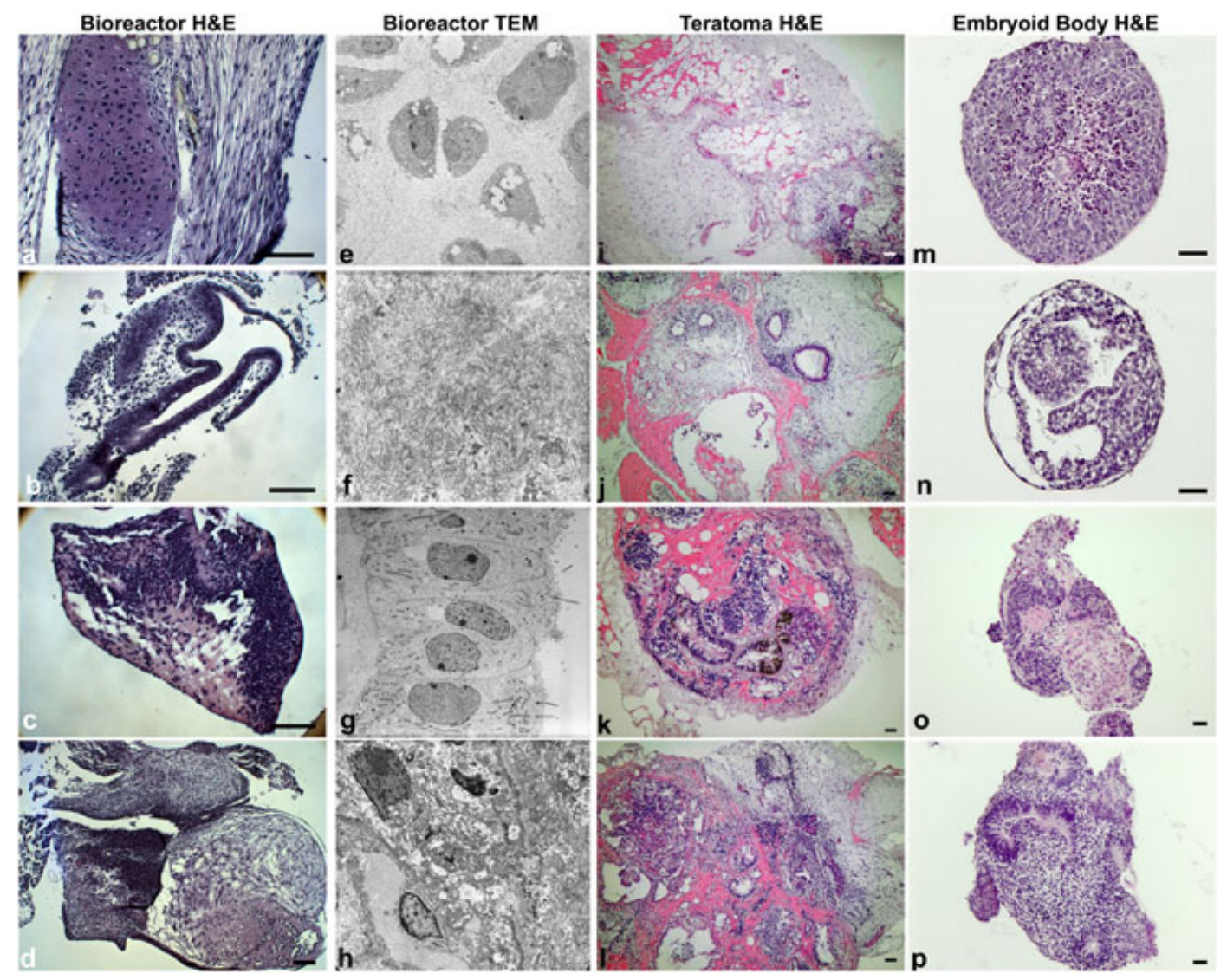

Figure 3. H\&E staining and electron microscopic analysis of tissue samples taken from bioreactors hESC-A, hESC-B and hESC-C, embryoid bodies and from in vivo teratomas. In samples from bioreactors hESC-A, -B and -C, a similar morphology was observed, showing that the hESCs differentiated and formed tissue-like structures. The morphology of these structures indicated differentiation into all three germ layers. Cartilage (a) and connective tissue (d) represented mesodermal, epithelial structures endodermal (b) and neuronal-like tissue (c) ectodermal differentiation (bar $=50 \mu \mathrm{m}$ ). In sections of the teratomas, in general predominantly mesodermal and endodermal structures but only few ectodermal structures could be detected: (i) connective tissue (mainly collagen-rich) and many adipocytes, (j) collagen-rich connective tissue, epithelial structures, adipose tissue, (k) pigmentary epithelial cells, epithelial structures forming some glandular structures, connective tissue interspersed with adipocytes, and (1) connective tissue and glandular structures (bar $=50 \mu \mathrm{m}$ ). Electron microscopic pictures of samples from the bioreactors show connective tissue (h) containing extracellular matrix with collagen ( $\mathrm{f}$ ), hyaline cartilage composed of mature chondrocytes surrounded by a collagen-rich extracellular matrix (e), and epithelial cells with kinocilia (g). In the embryoid bodies cultured for 6 days (m, n) and 20 days (o, p), only a limited number of differentiated structures could be observed. Furthermore, in a high percentage of the EBs, necrotic areas were visible $(\mathrm{m}, \mathrm{p} ; \mathrm{bar}=50 \mu \mathrm{m})$ 
than in the in vivo teratomas. This observation was also reflected by the results of differential gene expression analysis, where an enrichment for genes with a known role in neural differentiation could be observed in the bioreactor. These differences could be explained by influencing factors specific for the in vitro and in vivo environment, respectively. For example, it has been reported that one of the default pathways of spontaneous differentiation of hESCs in standard 2D culture conditions is neural differentiation (Reubinoff et al., 2001; Hornstein and Benvenisty, 2004). This observation is in accordance with the embryonic development in vivo, in which neurulation is the first step in organogenesis. The culture media and additives used could also have contributed to an enrichment of neural cell types in the bioreactors as compared to the teratomas. Especially bFGF, which is known to play an important role in neural development (Gremo and Presta, 2000; Dono, 2003) could be involved in these processes. It has been shown in vitro that bFGF has a strong mitogenic effect on neural stem and precursor cells (Hsu et al., 2007) and is also used as a differentiation factor in protocols for the neural differentiation of hESCs (Schwartz et al., 2008). Therefore spontaneous differentiation of the hESCs could have been influenced by the mitogenic and neurotophic activity of bFGF that resulted in an enrichment of neural cell types in the bioreactors. On the other hand the low percentage of ectoderm-derived tissues observed in the teratomas is in contrast to the results of other studies on teratoma formation of hESCs in mice, where a predominance of ectodermal and mesodermal differentiation is described (Gertow et al., 2004; Adewumi et al., 2007). One explanation could be the influence of the specific mouse model and the graft site, which has already shown to have an impact on the teratoma growth and differentiation pattern (Cooke et al., 2006).

Histological analysis of the EBs generated from the hESC cell line SA002, which was also used for bioreactor cultures, and an hiPSC cell line revealed only a limited
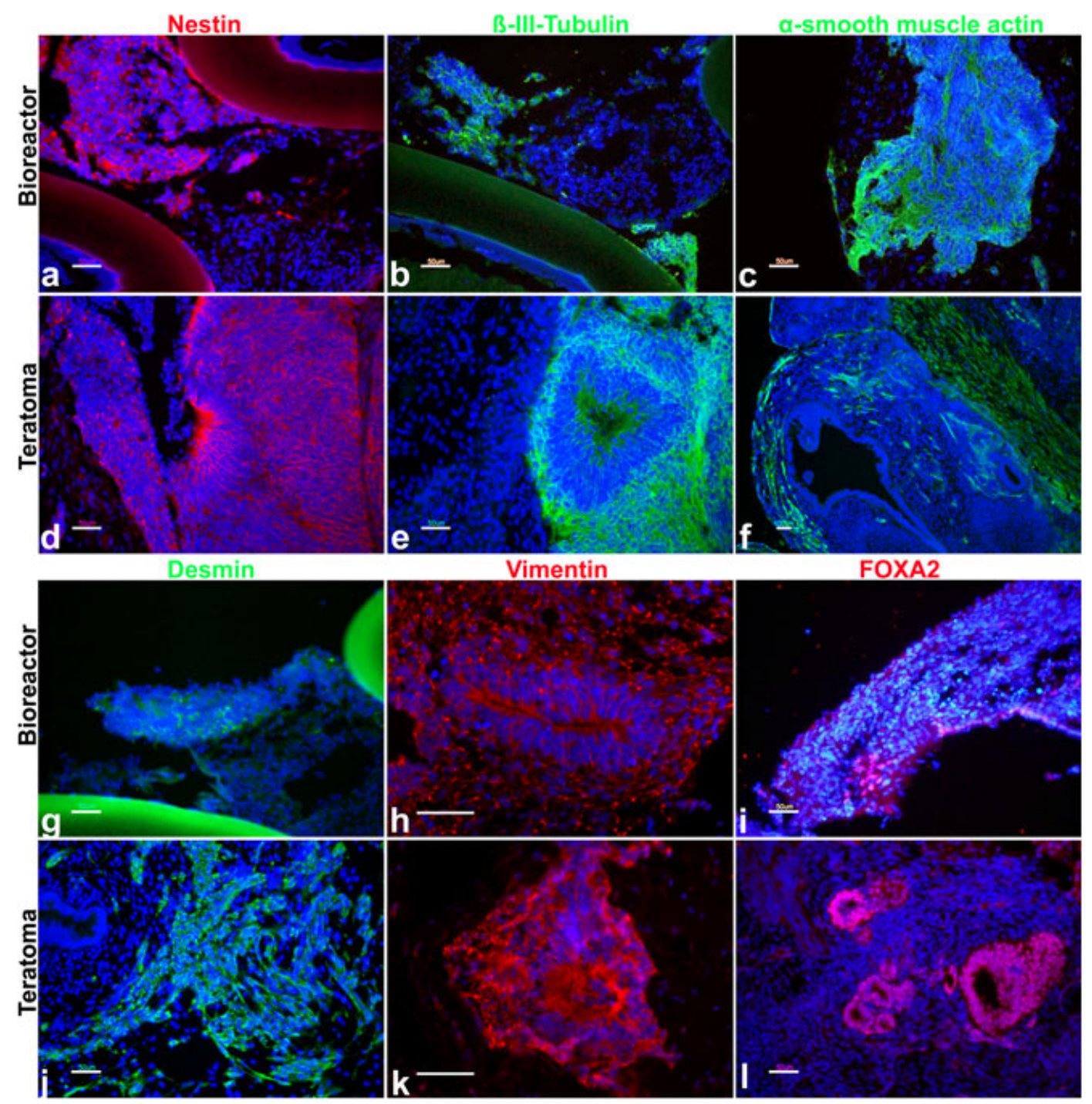

Figure 4. Immunofluorescence staining of tissue samples taken from the bioreactors hESC-A, -B and -C and in vivo teratomas: ectodermal markers nestin (a, d) and $\beta$ III-tubulin (b, e), mesodermal marker $\alpha$-smooth muscle actin (c, f), mesodermal markers desmin $(g, j)$ and vimentin $(h, k)$ and endodermal marker HNF-3 $\beta(i, 1)$. All samples are co-stained with DAPI (blue; bar $=50 \mu \mathrm{m})$ 


\section{H. Stachelscheid et al.}
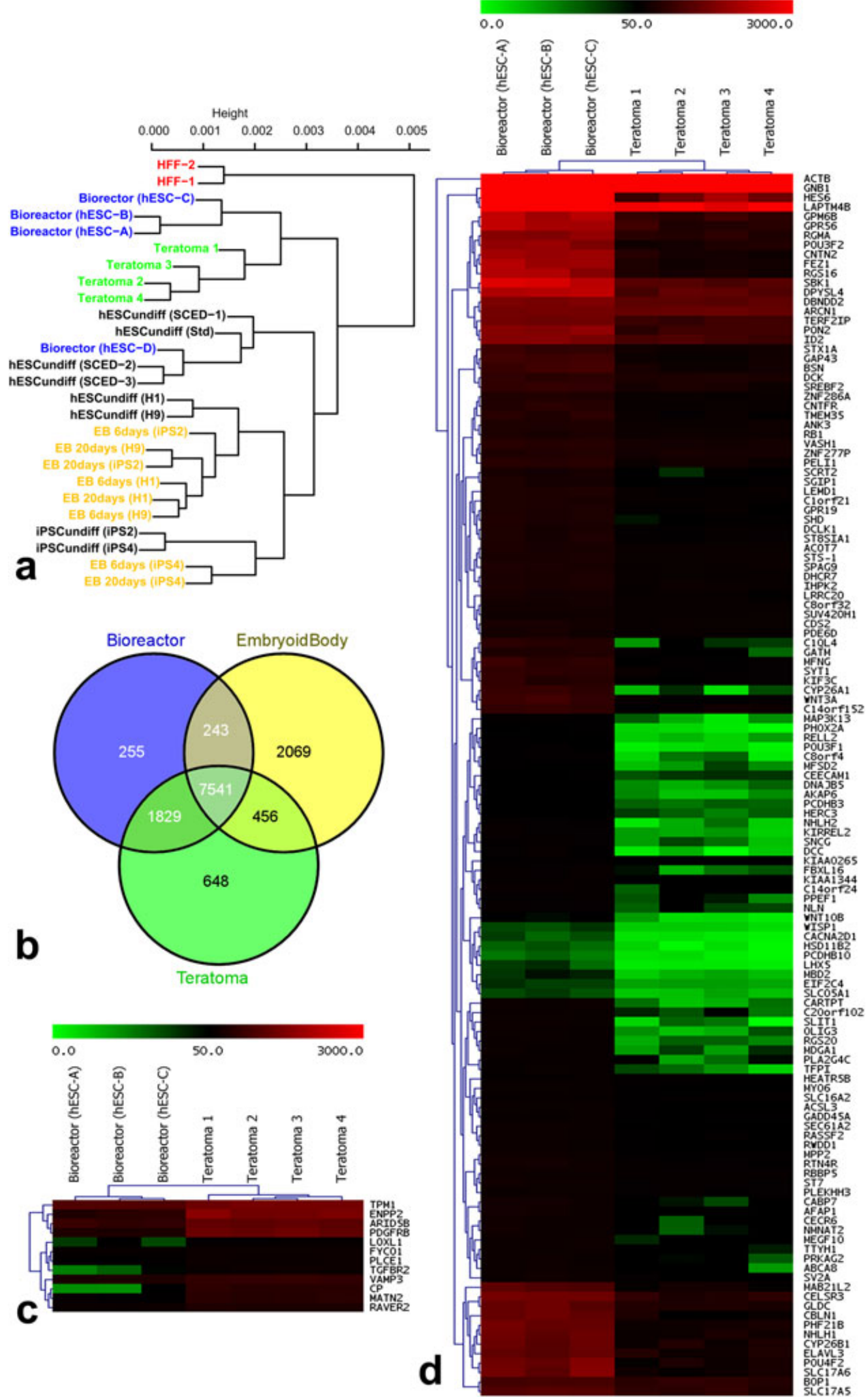

Figure 5. Hierarchical clustering and Differential gene expression. (a) Hierarchical clustering with average linkage clustering, showing that bioreactors hESC-A, -B and -C form a cluster and exhibit a similar expression profile to the teratomas. (b) Venn diagram comparing the expressed genes in bioreactors, teratomas and embryoid bodies. (c, d) The comparison of gene expression profiles of hESCs grown in bioreactors with those of teratomas was performed by significance analysis of microarrays (SAM) testing. The analysis resulted in 12 genes differentially expressed in the teratomas (c) compared to 146 genes in the bioreactors respectively (d). (b, c, are represented as heat maps clustered by hierarchical clustering of the samples and genes) 
number of morphologically distinct cell types and a much lower degree of differentiation and tissue-like organization. This limited degree of differentiation is also in accordance with previously published studies examining EB formation (Itskovitz-Eldor et al., 2000; Khoo et al., 2005; Pekkanen-Mattila et al., 2010). Gene expression analysis confirmed observations at the histological level, showing clustering of EBs together with undifferentiated cells. The differences observed between the undifferentiated cells and EBs of the cell lines SA002, H1, H9, iPS2 and iPS4 could represent an inter-cell line variability of expression of pluripotency genes (Adewumi et al., 2007) and differences in their in vitro differentiation capacity, as has been described for many pluripotent cell lines (Osafune et al., 2008).

Another observation that has also been described previously is necrotic areas in the centres of the EBs (Khoo et al., 2005; Pekkanen-Mattila et al., 2010). Comparable areas could not be detected in the bioreactors or teratomas and are most likely due to limited oxygen and nutrient transport within the EBs when they reach a certain size. These results indicate that by provision of dynamic mass exchange, the bioreactor much better reflects the in vivo situation, where it is supplied by the circulatory system of the host organism. This is supported by the results of our previous studies on mouse embryonic stem cell differentiation using the same bioreactor technology, where only a very low percentage of necrotic areas compared to mouse EBs could be observed (Gerlach et al., 2010a, 2010b).

The results of this study also indicate possible strategies to use the bioreactor for expansion of undifferentiated cells, regarding the utilization of feeder cells and medium composition. In the bioreactors hESC-A and hESC-B, which were inoculated with additional HFF feeder cells, the onset of differentiation indicated by the production of AFP and $\beta$-HCG was detected much later than in bioreactor hESC-C without feeder cells. This also shows that the bFGF in the used culture medium alone could not keep the cell in an undifferentiated state. This cytokine is known to promote the self-renewal of hESCs, both in co-cultures with MEF (Amit et al., 2000) and in feederfree cultures, when used in high concentrations (Xu et al., 2005a, 2005b). Thus, it can be hypothesized that after the feeder cell activity decreased, the used bFGF concentration was too low to sustain pluripotency and hESCs started to differentiate. To examine the influence of the culture medium on hESCs differentiation, a medium supplemented with a high concentration of FCS and no bFGF was used in bioreactor hESC-D, in which also no additional HFF feeder cells were inoculated. In this bioreactor the initially high activin A production decreased very slowly compared to all other reactors and reached a minimum after about twice the length of the time period observed in the bioreactors with feeder cells. Histological analysis revealed not only fibroblasts but also clusters of cells exhibiting a low cytoplasm:nucleus ratio, which is a typical feature of undifferentiated hESCs. In addition, many of these clusters co-expressed the pluripotency markers OCT4 and TRA-1-81, which suggests that these clusters represent undifferentiated hESCs. These results, together with the expression profile that showed a high correlation to hESC/HFF co-cultures, leads to the interpretation that differentiation in this reactor was suppressed by a 'feeder cell-like' activity. A possible explanation for the origin of this 'feeder cell-like' activity is that, under the influence of the culture medium used, some of the hESCs differentiated into cells exhibiting self-renewal supportive activity. This is in accordance with studies by other groups that showed that autologous hESC-derived feeder cells are able to support the self-renewal of undifferentiated hESCs (Xu et al., 2004; Stojkovic et al., 2005; Yoo et al., 2005; Gonzalez et al., 2008).

Based on these results, an approach to keep the hESCs in their undifferentiated state can be seen in the use of conditioned medium in the bioreactor. This could be addressed, for example, by an indirect co-culture achieved by interconnecting two bioreactors in a perfusion circuit. In this way, the cells are separated by the medium capillary membranes, while bidirectional exchange of soluble substances between the cells in the individual bioreactors is possible. Such a setup would also allow the use of active feeder cells that maintain their activity, in contrast to irradiated inactive feeder cells.

In conclusion, our results show that the bioreactor has the capacity to support differentiation of hESCs into 3D tissue structures that contain cells of all three germ layers. Cell structures formed in the bioreactor show a much higher degree of differentiation and organization than pluripotent cells differentiated in the form of EBs. These structures closely resemble teratomas developed in an in vivo environment at the histological, ultrastructural and RNA expression levels. The technology described could therefore be of interest for the development of alternatives to in vivo teratoma formation models for pluripotency assessment. The possibility of scaling down the bioreactor technology used (Zeilinger et al., 2011) allows reducing the costs of the system and facilitates the performance of larger numbers of parallel experiments. Furthermore, the method allows online control of the culture parameters and online monitoring of differentiation processes and could, therefore, be of interest for the development of tissue-engineering methods and embryotoxicity-testing assays. Since the bioreactor system supports the generation of differentiated cells, the technology could also be further developed for the production of specific cell types under 3D perfusion conditions in a closed system, by application of specific differentiation protocols. The latter would benefit from the scalability of the technology from laboratory to clinical translation or industrial application requirements.

\section{Acknowledgements}

This work was supported by the German Federal Ministry for Education and Research (BMBF) within the programme 'Cellbased Regenerative Medicine' (Grant No. 01GN0526, 01GN0528, 01GN0530) and by the European Commission (Grant No. STREPCT-2005-018940). We thank Alexander Ring, Wolfram Haider, Petra Schrade and Linda El-Ahmad for excellent help, technical assistance and fruitful discussions. 


\section{Author contributions}

H.S. designed and performed the study, performed and supervised the experiments, data and statistical analysis, interpreted data and wrote the manuscript; A.W.-G. and K.E. performed animal experiments; J.Je., J.E., M.R., R.S. and P.Bj. provided the cell line, performed experiments, evaluated experimental data and provided useful discussions; J.Jo., A.P. and J.A. performed microarray experiments; T.U. provided technical assistance during experiments and collected data; P.B. provided technical assistance during experiments, collected data and performed IHC experiments; K.Z. designed the study and interpreted data; and J.C.G. had the original idea for the project and developed the bioreactor technology. All authors commented on the manuscript.

\section{Supporting information on the internet}

The following supporting information may be found in the online version of this article:

Figure S1. Flow cytometric analysis of the cell suspensions inoculated into the bioreactors

Figure S2. Effect of different culture media and bFGF on HFFs

Figure S3. H\&E and immunofluorescence staining of tissue samples taken from bioreactors hESCs-D

Figure S4. H\&E staining of embryoid bodies derived from human iPSCs and cultured for different periods of time

Table S1. Samples used for expression profiling

Table S2. Result of functional annotation clustering using DAVID

\section{References}

Adewumi O, Aflatoonian B, Ahrlund-Richter L, et al. 2007, Characterization of human embryonic stem cell lines by the International Stem Cell Initiative. Nat Biotechno 25(7): 803-816.

Aleckovic M, Simon C. 2008; Is teratoma formation in stem cell research a characterization tool or a window to developmental biology? Reprod Biomed Online 17(2): 270-280.

Amit M, Carpenter MK, Inokuma MS, et al. 2000; Clonally derived human embryonic stem cell lines maintain pluripotency and proliferative potential for prolonged periods of culture. Dev Biol 227(2): 271-278.

Bar H, Strelkov SV, Sjoberg G, et al. 2004; The biology of desmin filaments: how do mutations affect their structure, assembly, and organisation? J Struct Biol 148(2): 137-152.

Beattie GM, Lopez $\mathrm{AD}$, Bucay $\mathrm{N}$, et al. 2005; Activin A maintains pluripotency of human embryonic stem cells in the absence of feeder layers. Stem Cells 23(4): 489-495.

Blum B, Benvenisty N. 2008; The tumorigenicity of human embryonic stem cells. Adv Cancer Res 100: 133-158.

Cooke MJ, Stojkovic M, Przyborski SA. 2006; Growth of teratomas derived from human pluripotent stem cells is influenced by the graft site. Stem Cells Dev 15(2): 254-259.

Desbaillets I, Ziegler U, Groscurth P, et al. 2000; Embryoid bodies: an in vitro mode of mouse embryogenesis. Exp Physiol 85(6): 645-651.

Dono R. 2003; Fibroblast growth factors as regulators of central nervous system development and function. Am J Physiol Regul Integr Comp Physiol 284(4): R867-R881.

Ellerstrom C, Strehl R, Noaksson K, et al. 2007; Facilitated expansion of human embryonic stem cells by single-cell enzymatic dissociation. Stem Cells 25(7): 1690-1696.

Gentleman RC, Carey VJ, Bates DM, et al. 2004; Bioconductor: open software development for computational biology and bioinformatics. Genome Biol 5(10): R80.

Gerlach JC, Hout M, Edsbagge J, et al. 2010a; Dynamic 3D culture promotes spontaneous embryonic stem cell differentiation in vitro. Tissue Eng Part C Methods 16(1): 115-121.

Gerlach JC, Lubberstedt M, Edsbagge J, et al 2010b; Interwoven four-compartment capillary membrane technology for three-dimensional perfusion with decentralized mass exchange to scale up embryonic stem cell culture. Cells Tissues Organs 192(1): 39-49.

Gerlach JC, Mutig K, Sauer IM, et al. 2003; Use of primary human liver cells originating from discarded grafts in a bioreactor for liver support therapy and the prospects of culturing adult liver stem cells in bioreactors: a morphologic study. Transplantation 76(5): 781-786.

Gertow K, Wolbank S, Rozell BR, et al. 2004; Organized development from human embryonic stem cells after injection into immunodeficient mice. Stem Cells Dev 13: 421-435.

Gilyarov AV. 2008; Nestin in central nervous system cells. Neurosci Behav Physiol 38(2): 165-169.

Gonzalez R, Loring JF, Snyder EY. 2008; Preparation of autogenic human feeder cells for growth of human embryonic stem cells. Curr Protoc Stem Cell Biol 1: units 1C 5.1-5.15.

Gremo F, Presta M. 2000; Role of fibroblas growth factor- 2 in human brain: a focus on development. Int J Dev Neurosci 18(23): 271-279.

Heins N, Englund MCO, Sjoblom C, et al. 2004; Derivation, characterization, and differentiation of human embryonic stem cells. Stem Cells 22(3): 367-376.

Hornstein E, Benvenisty N. 2004; The 'brainy side' of human embryonic stem cells. $J$ Neurosci Res 76(2): 169-173.

Hsu YC, Lee DC, Chiu IM. 2007; Neura stem cells, neural progenitors, and neurotrophic factors. Cell Transplant 16(2): 133-150.

Itskovitz-Eldor J, Schuldiner M, Karsenti D, et al. 2000; Differentiation of human embryonic stem cells into embryoid bodies compromising the three embryonic germ layers. Mol Med 6(2): 88-95.

James D, Levine AJ, Besser D, et al. 2005; $\mathrm{TGF} \beta /$ activin/nodal signaling is necessary for the maintenance of pluripotency in human embryonic stem cells. Development 132(6): 1273-1282.
Katsetos CD, Herman MM, Mork SJ. 2003a; Class III $\beta$-tubulin in human development and cancer. Cell Motil Cytoskel 55(2): 77-96.

Katsetos CD, Legido A, Perentes E, et al. 2003b; Class III $\beta$-tubulin isotype: a key cytoskeletal protein at the crossroads of developmental neurobiology and tumor neuropathology. $J$ Child Neurol 18(12): 851-866; discussion, 867.

Khoo ML, McQuade LR, Smith MS, et al. 2005; Growth and differentiation of embryoid bodies derived from human embryonic stem cells: effect of glucose and basic fibroblast growth factor. Bio Reprod 73(6): 1147-1156.

Kuhn K, Baker SC, Chudin E, et al. 2004; A novel, high-performance random array platform for quantitative gene expression profiling. Genome Res 14(11): 2347-2356.

Mizejewski GJ. 2004; Biological roles of alpha-fetoprotein during pregnancy and perinatal development. Exp Biol Med (Maywood) 229(6): 439-463.

Osafune K, Caron L, Borowiak M, et al. 2008; Marked differences in differentiation propensity among human embryonic stem cell lines. Nat Biotechnol 26(3): 313-315.

Pekkanen-Mattila M, Pelto-Huikko M, Kujala V, et al. 2010; Spatial and temporal expression pattern of germ layer markers during human embryonic stem cell differentiation in embryoid bodies. Histochem Cell Biol 133(5): 595-606.

Prigione A, Adjaye J. 2010; Modulation of mitochondrial biogenesis and bioenergetic metabolism upon in vitro and in vivo differentiation of human ES and iPS cells. Int $J$ Dev Biol 54(11-12): 1729-1741.

Prigione A, Fauler B, Lurz R, et al. 2010; The senescence-related mitochondrial/oxidative stress pathway is repressed in human induced pluripotent stem cells. Stem Cells 28(4): 721-733.

Przyborski SA. 2005; Differentiation of human embryonic stem cells after transplantation in immune-deficient mice. Stem Cells 23 (9): 1242-1250.

Reubinoff BE, Itsykson P, Turetsky T, et al. 2001; Neural progenitors from human embryonic stem cells. Nat Biotechnol 19(12): 1134-1140. 
Saeed AI, Sharov V, White J, et al. 2003; TM4: a free, open-source system for microarray data management and analysis. Biotechniques 34(2): 374-378.

Schwartz PH, Brick DJ, Stover AE, et al. 2008; Differentiation of neural lineage cells from human pluripotent stem cells, Methods 45(2): 142-158.

Stenman UH, Tiitinen A, Alfthan $\mathrm{H}$, et al. 2006; The classification, functions and clinical use of different isoforms of HCG. Hum Reprod Update 12(6): 769-784.

Stojkovic P, Lako M, Stewart R, et al. 2005; An autogeneic feeder cell system that efficiently supports growth of undifferentiated human embryonic stem cells. Stem Cells 23(3): 306-314.

Valbuena D, Galán A, Sánchez E, et al. 2006; Derivation and characterization of three new Spanish human embryonic stem cell lines (VAL-3 -4 -5) on human feeder and in serum-free conditions. Reprod Biomed Online 13(6): 875-886.
Vallier L, Alexander M, Pedersen RA. 2005; Activin/Nodal and FGF pathways cooperate to maintain pluripotency of human embryonic stem cells. $J$ Cell Sci 118(pt 19): 4495-4509.

Vallier L, Pedersen RA. 2005; Human embryonic stem cells: an in vitro model to study mechanisms controlling pluripotency in early mammalian development. Stem Cell Rev 1(2): 119-130.

Xiao L, Yuan X, Sharkis SJ. 2006; Activin A maintains self-renewal and regulates fibroblast growth factor, Wnt, and bone morphogenic protein pathways in human embryonic stem cells. Stem Cells 24(6): 1476-1486.

Xu C, Jiang J, Sottile V, et al. 2004; Immortalized fibroblast-like cells derived from human embryonic stem cells support undifferentiated cell growth. Stem Cells 22(6): 972-980.

Xu C, Rosler E, Jiang J, et al. 2005a; Basic fibroblast growth factor supports undifferentiated human embryonic stem cell growth without conditioned medium. Stem Cells 23(3): 315-323.

Xu RH, Chen X, Li DS, et al. 2002; BMP4 initiates human embryonic stem cell differentiation to trophoblast. Nat Biotechnol 20(12): 1261-1264.

Xu R-H, Peck RM, Li DS, et al. 2005b; Basic FGF and suppression of BMP signaling sustain undifferentiated proliferation of human ES cells. Nat Methods 2(3): 185-190.

Yoo SJ, Yoon BS, Kim JM, et al. 2005; Efficient culture system for human embryonic stem cells using autologous human embryonic stem cell-derived feeder cells. Exp Mol Med 37(5): 399-407.

Zeilinger K, Schreiter T, Darnell M, et al. 2011; Scaling down of a clinical threedimensional perfusion multicompartment hollow fiber liver bioreactor developed for extracorporeal liver support to an analytical scale device useful for hepatic pharmacological in vitro studies. Tissue Eng Part C Methods 17(5): 549-556. 